\title{
Los estándares como instrumentos politicos: ciencia y Estado franquista a finales de los años cincuenta $^{1}$
}

\author{
LINO CAMPRUBí \\ UCLA \\ linocamprubi@ucla.edu
}

Recibido: 24.05 .2009

Aceptado: 02.11.2009

\section{INTRODUCCIÓN HISTORIOGRÁFICA}

Las páginas que siguen narran la breve historia de Punto Azul, un proyecto de estandarización de elementos hormigonados de construcción —en principio, viguetas, vigas cortas cuya función estructural principal es formar parte de un forjado horizontal o inclinado que de lugar a un suelo, un techo o una rampafabricados en serie. Punto Azul se ideó en 1958, vio la luz en 1959 y fue definitivamente cancelado en 1960. Indagar en los diversos aspectos de este proyecto truncado y del que sólo quedan menciones en los archivos servirá para explorar un tema de mucha mayor amplitud: el de las relaciones de la historia de las ciencias y las técnicas con la historia de los Estados.

La historia de las ciencias solía concebirse tradicionalmente como una historia de los principales descubrimientos científicos, de las grandes teorías de la ciencia (el ejemplo clásico es Koyré, 1939). Correspondientemente, la historia de las técnicas aparecía como historia de innovaciones, y en muchas ocasiones simplemente de las aplicaciones de teorías científicas (Kline, 1995). En el último tercio del siglo XX, dicha perspectiva cambió de la mano del análisis sociológico de las ciencias — a menudo, pero no siempre, de raigambre marxista— aten-

1 Agradezco los comentarios a borradores de este artículo a muchas personas, en especial a Soraya de Chadarevian y Fernando Guirao, así como a Amy Slaton, Wiebe E. Bijker y el resto de los asistentes al panel «A Concrete Mediterranean: Engineering, Cement and Society in the European Periphery (20 ${ }^{\text {th }}$ and $21^{\text {st }}$ centuries)» SHOT, Lisboa 2008. Agradezco también al departamento de publicaciones del Instituto Eduadro Torroja de las Ciencias de la Construcción y, sobre todo, a su directora, Virtudes Azorín, todas las facilidades y sugerencias prestadas. Asimismo, es de agradecer la lectura atenta que los dos árbitros de Empiria dedicaron a estas páginas. 
to sobre todo al contexto de los descubrimientos científicos (Kuhn, 1962; Huerga, 1999). Como extensión natural de esta perspectiva, no faltaron los intentos de reducción de las ciencias a sus contextos sociológicos, pero algunos historiadores de la ciencia se esforzaron - muchas veces sin éxito, dado lo arduo de la empresa- en mostrar que sus mejores frutos vendrían de entender cómo partes 'externas' a una ciencia podían convertirse en 'internas' a ella, sufriendo así una transformación (para el programa fuerte sociologista, Barnes y Bloor, 1982; para la idea de transformación, Bueno, 1992-93: 301-303; para un debate acerca del reduccionismo sociologista, Bloor, 1999 y Latour, 1999).

Mientras los historiadores de las ciencias investigaban los contextos de la génesis de éstas, a menudo olvidaron preguntarse, probablemente porque en ocasiones se dejaron arrastrar por el relativismo, cómo las ciencias a su vez afectaban a las sociedades a las que se aplicaban. En cambio, los historiadores económicos, en especial los estudiosos del siglo XX, enfatizaron el papel de la investigación científica, pero su perspectiva implicaba casi siempre dar los resultados de aquella por supuestos (Kondratieve, 1988; Mokyr, 1990). Una minoritaria pero creciente historiografía de las técnicas y las ciencias intenta salvar este bache; al igual que el presente estudio, ésta toma a menudo el Estado como unidad de análisis, dado que de él han dependido en el siglo XX gran parte de los fondos para investigación científica y técnica, y dado que a él se han referido muchos proyectos ideados por investigadores (Hughes, 1990; Picon, 1992; Alder, 1997; Usselman, 2002).

Si las relaciones de las diferentes ciencias con diferentes sociedades legitiman el estudio de la historia de la ciencia como herramienta para el estudio del Estado, es porque la historia de la ciencia no es sólo la historia de grandes teorías científicas, sino la historia de los objetos físicos con que los científicos trabajan (Hacking, 1983; Bueno, 1992-93; Roberts et al., 2007). Esto permite al historiador perseguir a los objetos científico-técnicos desde su transformación en el laboratorio hasta su implantación en áreas, en principio, externas al laboratorio (Latour, 1988). Esto permite también seguir a los objetos productos del laboratorio en su expansión geográfica, lo cual da fundamento material a la universalidad de muchos contenidos científicos — su necesidad es un problema filosófico distinto-, aunque a veces se confundan ambos (O'Connell, 1993; Wise, 1995; Álvarez; 2004).

El anterior argumento afecta de modo especial a los estándares internacionales, los cuales nos ocuparán en posteriores secciones. Frente a las tesis, provenientes sobre todo de algunas corrientes de historia económica, tendentes a considerar a los estándares como meros resultados naturales del crecimiento capitalista (Chandler y Takashi, 1990), atender al detalle de su nacimiento e implantación supone en muchas ocasiones situarse en los bordes de las conexiones de la ciencia y la política.

El presente trabajo aspira a estudiar ciertos objetos científico-técnicos como instrumentos políticos. Para ello, veremos cómo determinados objetos fisicalistas, aparentemente tan anodinos desde el punto de vista socio-político como las 
viguetas de hormigón pretensado fabricadas en serie, pueden encarnar proyectos político-económicos de envergadura (para una interpretación política de un objeto técnico ya clásica, Winner, 1986). El intento no merecería el esfuerzo si fuera incapaz de aportar perspectivas nuevas a las historias estrictamente políticas o económicas al uso (para la conexión entre técnica y nación-Estado en países periféricos en producción científica, ver Carroll, 2006 y Saraiva, 2007). Para ello, hay que concebir a la política, a nivel estatal, no como la mera sucesión de regímenes o gobiernos, sino como un campo complejo en el que además de gobernantes y gobernados, interaccionan -mediante objetos físicos- agentes económicos así como agentes pertenecientes a otros Estados, sin los cuales la historia política se arriesga a perder el pie en las sociedades que estudia ${ }^{2}$.

Es importante destacar las peculiaridades que tiene la aplicación del esquema metodológico recién presentado a la España del tránsito del llamado primer franquismo a la etapa posterior. Los años que van desde la crisis y cambio de gobierno de 1957 hasta la puesta en marcha del Plan de Estabilización en 1959 suelen narrarse como una época de cambio profundo dentro del régimen de Franco (Gallo, 1969; Anderson, 1970; Moradiellos, 2000). Cargos públicos de alta preparación técnica habrían desviado el rumbo de la nave autárquica hacia la economía de mercado y la integración europea mediante un golpe de timón liberalizador. Veremos en este artículo que esta imagen queda matizada cuando se aplica a ella la metodología arriba expuesta; pero ahora conviene centrarse en el papel que ella ha otorgado a la ciencia del primer franquismo, que es prácticamente nulo. En efecto, la mayoría de los historiadores de la ciencia en España pasan por los años del primer franquismo como por un callejón oscuro y suelen dar por supuesto que, bajo el 'totalitarismo', la investigación científica y técnica queda ahogada (Sanz, 1997; Sánchez Ron, 1999). Al contrario que ocurre en otros contextos, al estudiar regímenes no democráticos gran parte de la historiografía de la ciencia sigue viendo el contexto social como influencia 'externa' a las ciencias, y no como la fuente de los materiales de que ésta se nutre. Si bien es cierto que esta anomalía ha sido revisada por la historiografía de otros países, en España esto se hace más difícil por su consideración de país periférico en cuestiones científicas (Blackbourne, 2006; Augustine, 2007). Sólo algunos estudios de caso han desafiado esta situación (Roca i Rosell y Sánchez-Ron, 1990; Ordóñez y Sánchez-Ron, 1996; Santesmases, 2001; Presas i Puig, 2005). En la conclusión volveremos sobre estos dos asuntos —el giro político y el lugar de la ciencia en el primer franquismo- para evaluar hasta qué punto la metodología seguida transforma este tratamiento de las ciencias y las técnicas durante el primer franquismo.

2 «Cabría decir, por tanto, que las ideas se hacen presentes no como «contenidos de una mentalidad», sino como las formas mismas de los automóviles que se utilizan (o se desea destruir), de las lámparas que alumbran, de los materiales con los cuales se construyen las autopistas, las casas, los trajes, y hasta los alimentos y las medicinas. Estas son las 'ideas' que mueven a la historia, éstas son las ‘superestructuras’ o, por lo menos, allí están como principios activos» (Bueno, 1991: 161; Bueno, 1972).

EMPIRIA. Revista de Metodología de Ciencias Sociales. N. ${ }^{\circ}$ 18, julio-diciembre, 2009, pp. 85-114. ISSN: 1139-5737 
En el presente artículo, se ofrece el contexto en el que debe enmarcarse la corta trayectoria de Punto Azul y las controversias generadas en torno a ella (apartado primero). En lugar de ser una solución técnica obvia y neutral, Punto Azul encarnaba un proyecto de gran alcance político-económico. Más precisamente, la historia de Punto Azul desvela tres líneas principales de este proyecto: la industrialización de la construcción española (apartado segundo), la integración de España en las incipientes redes de colaboración e intercambio europeas (apartado tercero) y la regulación estatal de la empresa privada (apartado cuarto). En la conclusión se ofrecerán algunos de los resultados que la perspectiva propuesta en este artículo arroja cuando se aplica al estudio de Punto Azul.

\section{PUNTO AZUL Y EL INSTITUTO TÉCNICO DE LAS CIENCIAS DE LA CONSTRUCCIÓN}

Punto Azul era, ante todo, un proyecto de regulación de productos. Consistía en un logotipo azulado que iría estampado en elementos estructurales de construcción como medio de certificar que cumplían ciertos estándares mínimos de calidad y seguridad. En principio, se limitaría a experimentar con una norma provisional para viguetas de hormigón pretensado, uno de los primeros elementos al que se había aplicado la nueva técnica y cuyo uso se había propagado entre los fabricantes españoles, dada la mayor solidez que daba a las estructuras con precios más bajos. También se limitaría a elementos fabricados en serie, punto, como veremos, de especial importancia. El proyecto surgió del Instituto Técnico de la Construcción y del Cemento (ITCC) en 1958 y en 1960 fue abortado por el Patronato Juan de la Cierva del Consejo Superior de Investigaciones Científicas (CSIC), al que el ITCC pertenecía. Aunque los impulsores de Punto Azul defendieron el proyecto alegando su naturaleza puramente técnica, veremos que éste se entiende mejor dentro de un programa de más largo alcance para la transformación de la economía política del país, a saber, la industrialización de la construcción.

En el logotipo de Punto Azul figuraban las siglas ITCC pintadas en color azul. El ITCC resultó del desarrollo de una iniciativa privada sin ánimo de lucro por parte de ingenieros y arquitectos de prestigio que, en 1934, durante la Segunda República española, fundaron el Instituto de la Construcción (IC). Tras la Guerra Civil, algunos de estos ingenieros pasaron a ocupar puestos destacados en el nuevo gobierno, incluyendo a Alfonso Peña Boeuf, Ministro de Obras Públicas, y Eduardo Torroja Miret, Delegado de Cemento y colaborador de Antonio Suanzes, Ministro de Industria en dos ocasiones, director del Instituto Nacional de Industria y presidente desde 1942 del Patronato Juan de la Cierva. Tales posicionamientos en torno al poder político por parte de ingenieros civiles y militares no eran fruto de meras coincidencias, sino que representaban la cristalización de importantes sectores en el seno del nuevo régimen que lo consideraban como una oportunidad de implantar en España la racionalidad y eficiencia propias de su profesión (Peña Boeuf, 1954: 228-243). A juzgar por la suerte del IC, 
no andaban del todo errados en sus cálculos quienes así pensaban. En efecto, Torroja, ya entonces internacionalmente famoso por sus estructuras hormigonadas y que tomó el mando del IC tras la Guerra, logró no sólo que éste pasase a formar parte del CSIC, sino que, mediante su fusión con el Instituto del Cemento en 1949 (por la que el IC pasó a llamarse Instituto Técnico de la Construcción y del Cemento) y el cobro de cánones por ventas de cemento y carbón así como de créditos especiales, se convirtiese el nuevo ITCC entre 1949 y 1953 - año de la inauguración del laboratorio del ITCC en Madrid, el famoso Costillares-en el mejor financiado de los Institutos del Patronato Juan de la Cierva, que era a su vez, y con distancia, el mejor financiado de cuantos componían el CSIC (López García, 1994; para un repaso por las principales líneas de investigación del ITCC, Azorín et al., 2005). Para comprender el peso político de los líderes del ITCC, basta decir que el canon por ventas de cemento, junto con préstamos especiales, lo negoció Torroja personalmente con el General Francisco Franco 3 .

Desde su comienzos, el objetivo del IC, y después del ITCC, fue el de industrializar la construcción en España, al modo como se habían industrializado otras ramas de la producción económica (aunque con retraso respecto a economías más industrializadas; Arribas, 1994: 156). Esto significaba, ante todo, la sustitución de producción artesana por maquinaria o por pasos mecánicos y previamente fijados para los operarios. La industrialización de la construcción permitiría la plena racionalización de todo tipo de obras, entendida ésta como la búsqueda de la máxima eficiencia para cada problema determinado mediante un diseño apropiado y una mecanización de la producción de los materiales y de los modos y técnicas de trabajo. Este plan, pese a su aparente restricción al campo de la construcción, incluía tomas de partido respecto al papel del trabajador (en tanto se perseguía mecanizar su labor) y de la empresa privada y pública (principales consumidores, y por tanto artífices, de los métodos de industrialización de la construcción). Era deudor, en fin, de una determinada visión de la economía política española y del papel de la ingeniería en su «modernización».

Una de las actividades principales del ITCC consistía en ensayar materiales y estructuras para particulares, tanto consumidores como productores. Dependiendo de la prueba requerida, los ensayos eran de carácter matemático (cálculo), físico (mecánica), químico (purezas), o experimental (modelos) ${ }^{4}$. Desde principios de los años cincuenta, el número de peticiones para ensayos de este tipo se

\footnotetext{
${ }^{3}$ Instituto Nacional de Industria, Archivo SEPI, Madrid. 0000024888: Eduardo Torroja, «Carta a Antonio Suanzes», 1950-1951.

4 También se hacían ensayos in situ de estructuras ya construidas por medio de "laboratorios móviles', furgonetas con diseñadas para llevar los métodos y el equipamiento del ITCC a todo el territorio nacional. Las pruebas de los 'laboratorios móviles' incluían estudios esclerométricos (miden la uniformidad del hormigón), ultrasónicos, radiográficos, de deformaciones, acústicos y otros. Por supuesto, los ensayos en el laboratorio del ITCC eran más completos, pues podían incluso incluir modelos para probar resistencia, forma, etc. Diversos autores han señalado la importancia de los modelos en ingeniería, en especial para la relaciones del laboratorio con la realidad exterior de las que hemos hablado en la introducción de este artículo (Bijker, 2005).
}

EMPIRIA. Revista de Metodología de Ciencias Sociales. N. ${ }^{\circ}$ 18, julio-diciembre, 2009, pp. 85-114. ISSN: 1139-5737 
multiplicó. El volumen era tal que amenazaba con sobrecargar los recursos, limitados, del ITCC — recordemos que un exceso de ensayos individuales había sido-, en 1918, una de las principales causas del cierre del uno de los centros de investigación científica y técnica más importantes de la época, el alemán $P h y$ -

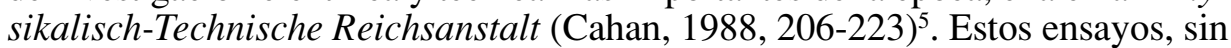
embargo, constituían uno de los principales puntos de conexión del Instituto con la empresa privada; no había, pues, que suspenderlos, sino armonizarlos con los programas de más largo alcance del ITCC, en especial la industrialización de la construcción. El modo de lograr esta armonía y de explotar una oportunidad única de incidir en la empresa privada era la sistematización de los ensayos ${ }^{6}$. Para ello, Torroja concibió Punto Azul.

En apartados posteriores analizaremos las circunstancias del nacimiento de Punto Azul y las razones de su acortada trayectoria. Ahora procede evaluar la defensa que de él hacían los promotores del proyecto, que repetían que «Punto Azul era una solución técnica basada en consideraciones técnicas para desempeñar una función de investigación técnica para la industria con el menor esfuerzo e inversión posible» ${ }^{7}$. Como quedó anunciado en la introducción, pese a estos intentos de presentar Punto Azul como un asunto puramente técnico, lo cierto es que éste sólo era evidente para sus padrinos, dado que estaba estrechamente vinculado al proyecto de industrialización de la construcción que abanderaban los directivos del ITCC. En efecto, Punto Azul, como certificado de mínimos de calidad para productos fabricados en serie, era un modo de promocionar la prefabricación y la estandarización, guiadas ambas por las «ciencias de la construcción ${ }^{8}$. Para que la industrialización de la construcción fuera

5 Por ejemplo, entre 1958 a 1960 los técnicos del ITCC ensayaron individualmente 600 viguetas de hormigón para un solo fabricante. Archivo Histórico del Instituto de las Ciencias de la Construcción Eduardo Torroja, Madrid (en adelante, AHT): AHT/N/A/040/001, «Certificado de Echegaray y Comba», 22 de noviembre 1960.

6 Según Judy Klein, con anterioridad a la Segunda Guerra Mundial, la empresa Bell desarrolló en EEUU métodos estadísticos de ensayo. Durante la Guerra, y debido a la necesidad de probar armamento a gran velocidad, importantes economistas al servicio de los ejércitos perfeccionaron esos métodos estadísticos. Una vez terminada la Guerra, empresas privadas dedicadas a la producción en masa, sobre todo japonesas (de la mano de Williams Edwards Deming), recogieron el testigo y desarrollaron aún más los métodos de ensayo sistemático (Klein, 2000: 57). El ITCC incorporó los métodos estadísticos para sus ensayos sistemáticos y Torroja fue pionero en la aplicación de la probabilidad a los análisis de seguridad del hormigón (Cowan, 1978: 93).

7 AHT/N/A/043/001, «Nota referente a los ensayos a que dará lugar la supresión de Punto Azul», 23 de octubre de 1960: 1.

8 Asier Salas ha reconocido la importancia del ITCC para la normalización de las técnicas constructivas en España, pero sin subrayar la prefabricación, la investigación científica, el uso de materiales nuevos, ni la cristalización de estas tres líneas de trabajo en el proyecto de Punto Azul (Salas, 2004: 130-31). Los estudios de María José Casinello también son obligados para comprender los contextos más técnicos de la normalización impulsada por el ITCC (Casinello, 1997; Casinello, 2000). Ambos estudios insisten en la importancia que en estos procesos tuvo la creciente demanda de vivienda económica en los núcleos urbanos, asunto estudiado especialmente por Carlos Sambricio (Sambricio, 2004).

EMPIRIA. Revista de Metodología de Ciencias Sociales. N. ${ }^{\circ}$ 18, julio-diciembre, 2009, pp. 85-114. ISSN: $1139-5737$ 
posible, todos los elementos de una obra habían de poder distribuirse desde el laboratorio o el taller, esto es, habían de someterse a las «ciencias de la construcción», la estandarización, y la prefabricación. En este sentido, el hecho de que se centrara en elementos estructurales de hormigón pretensado no es casual, pues este material estaba estrechamente ligado a esos tres elementos, veamos cómo.

\section{HORMIGÓN PRETENSADO E INDUSTRIALIZACIÓN: PREFABRICACIÓN, ESTANDARIZACIÓN Y «CIENCIAS DE LA CONSTRUCCIÓN»}

El uso del hormigón pretensado no se popularizó hasta la década de los cuarenta del siglo XX. Mientras que el hormigón armado había sido de uso común en la primera mitad del siglo $\mathrm{XX}$, el hormigón pretensado fue prácticamente desconocido hasta 1928, cuando el ingeniero francés Eugène Freyssinet volvió a su estudio y determinó varias características necesarias para su correcto funcionamiento - en especial la calidad del hormigón y la capacidad tensora del acero-, ambas superiores a las habituales (Levi, 1951: 219-225). Al igual que el hormigón armado, el pretensado utilizaba el acero para dotar de mayor resistencia y durabilidad a los elementos de hormigón, de modo que pudieran utilizarse como elementos estructurales en diversas construcciones. Pero mientras que el hormigón armado resultaba de la adición del hormigón a moldes de acero ya en la obra y laboriosos encofrados de madera que exigían mano de obra muy especializada, el hormigón pretensado combinaba hormigón y acero antes de recibir cargas externas en un proceso más complejo que normalmente requería trabajo en el taller: se tensaban finos cables de acero y se colocaban en un molde; se rellenaba el molde con hormigón, al cual se fijaba el acero mediante anclajes u otros dispositivos; $y$, finalmente, se relajaba la tensión de los cables de modo que la pieza en su totalidad sufría pre-compresión. Esta técnica neutralizaba tracciones y fuerzas tensoras una vez que la pieza estructural quedaba colocada, de modo que el resultado era un elemento duradero capaz de resistir cargas y presiones elevadas incluso para largas distancias entre puntos de apoyo (luces). La adherencia del hormigón a los cables de acero transformaba a los dos materiales en una única pieza; creaban, en palabras de Torroja, un nuevo material, constituyendo, así, «la más fina y compleja de la invenciones actuales en materiales de la construcción». (Torroja, 1958) ${ }^{9}$.

\footnotetext{
9 Torroja cita a D. J. M. Zafra, uno de los introductores del hormigón armado en España y predecesor de Torroja en la Universidad: «el acero da fibra a las piedras y el hormigón da masa al hierro». Aunque Zafra aplicaba su frase al hormigón armado, Torroja la consideraba más ajustada al pretensado. Hay que señalar aquí que «hormigón pretensado» iba, y aun va, referido a dos técnicas diferentes: el pretensado estricto, más afín a la prefabricación en taller, y el post-tensado, para el cual los cables se tensaba una vez vertido el hormigón al molde en la obra. Conviene también destacar que Torroja no pensaba que el «nuevo material» fuera a reemplazar a todos los predecesores, sino que cada situación requeriría un material apropiado: «esto ha ocurrido a menudo en las técni-
}

EMPIRIA. Revista de Metodología de Ciencias Sociales. N. ${ }^{\circ}$ 18, julio-diciembre, 2009, pp. 85-114. ISSN: 1139-5737 


\section{Hormigón pretensado y prefabricación}

Una de las razones por las que el hormigón pretensado merecía la alabanza de Torroja era que favorecía la prefabricación: «el pretensado y la prefabricación se han impulsado mutuamente» (Torroja, 2004: 78). Dado que el material exigía el trabajo en taller, era posible producir elementos pretensados en condiciones totalmente controladas. Esto no solamente permitía un uso más controlado de los materiales y un trabajo más disciplinado y organizado, sino que, además, abría la posibilidad a la producción en masa de dichos elementos. Según Torroja, el ingeniero debía estar igualmente atento a la economía que a la estética ${ }^{10}$. Y la prefabricación e incluso la producción en masa o en serie no estaban reñidas, en el caso del pretensado, con la atención a la forma estética, por la que Torroja ha pasado a la historia de las estructuras. Un diseñador podía construir estructuras originales con elementos idénticos, siempre y cuando fuera capaz de «salirse de los clásico y lo tradicional con su ingenio e inventiva» (Torroja, 2004: 85).

Es importante percibir la división del trabajo que subyacía a esta concepción de la construcción: mientras el trabajo artesano debía ser sustituido por trabajo mecánico, el genio del diseñador había de salvarse de la mecanización; una perspectiva parecida a la que ya habían adoptado los ingenieros con carrera universitaria que popularizaron el hormigón armado en Estados Unidos en la primera mitad del siglo XX (Slaton, 2001; esta estrategia se ha llamado «autoridad mecánica», Alder, 1997). El ingeniero no sería meramente un experto, aunque veremos que habrá de ser considerado científico, sino que el genio y la intuición formarán parte de su labor. Torroja, además, justificaba esta división del trabajo inherente a la industrialización que el ITCC promovía como una labor 'cristiana' dirigida a liberar a cada vez más gente de trabajos no cualificados para que pudieran realizar su potencial humano, aunque no se detenía en explicar los muchos obstáculos prácticos que tal aspiración parece oponer (Torroja, 1959).

\section{Hormigón pretensado y estandarización}

La prefabricación, y muy especialmente la producción en masa, no sólo permitía, sino que implicaba, la estandarización. La producción en masa exigía estándares de diseño, en los que la forma de la pieza ha de darse especificada

cas; los coches no han sustituido a los carros y los aviones no han hecho disminuir el número de coches; sin embargo, los carros no son buenos para la velocidad y los coches no son buenos para volar» (Torroja, 1958: 25).

10 «Es el ingeniero, en tanto ingeniero, quien ha de buscar las ventajas económicas; porque es normal que en la construcción la economía no dependa sólo de las dimensiones sino de un cambio total de los procesos de construcción.» (Torroja, 1958: 28).

EMPIRIA. Revista de Metodología de Ciencias Sociales. N. ${ }^{\circ}$ 18, julio-diciembre, 2009, pp. 85-114. ISSN: 1139-5737 
para asegurar su compatibilidad funcional con otras piezas (por ejemplo, el tamaño de las viguetas repercute en el de las edificaciones). Este proceso había ya tenido lugar en diversas líneas de producción industrializadas, tales como cables para telégrafos o partes de coches producidas en una cadena de montaje. ${ }^{11}$ Entre las ventajas de este modo de producción estaba la de asegurar la demanda antes de invertir en equipos para producir en serie; según Torroja, «sólo mediante la normalización pueden obtenerse todas las ventajas de la industrialización» (Torroja, 1958: 32).

Más acuciantes que los estándares de diseño eran los estándares de rendimientos mínimos, que no definen la forma de las piezas sino sus características funcionales mínimas (Timmermans \& Berg, 2003). En el caso del hormigón pretensado, estos incluyen no sólo la pieza final sino sus componentes, tales como el cemento, el agua o el acero. La urgencia de estos estándares venía dada porque el uso del hormigón pretensado se había expandido muy rápidamente tras el vencimiento de las patentes de Freyssinet en la Europa de los años cuarenta (durante la Guerra, Alemania permitió la violación de estas patentes dada su carestía de acero), ávida de materiales para su reconstrucción (Grote y Marrey, 2000: 4384). Pero esta expansión estuvo acompañada de falta de experiencia, de condiciones de uso, de recomendaciones, de especificaciones o de instrucciones. La estandarización del pretensado era la vía para salir de la «dispersión existente en el producto», en palabras de técnicos del ITCC.

\section{Hormigón pretensado e investigación científica}

¿De qué forma podían implantarse esos estándares tan necesarios? A juicio de los miembros del ITCC, mediante la determinación científica de las variables de las que dependía el buen funcionamiento del hormigón pretensado. Desde finales del siglo XIX, la estandarización ha sido una de las principales actividades no sólo de ingenieros, sino de físicos, químicos y otros científicos (Hunt, 1994; Rusell, 2006). No es de extrañar, por tanto, que el proyecto político-económico de industrializar la construcción fuera unido al proyecto científico de constitución de las «ciencias de la construcción.» Torroja reconocía que la industrialización de la construcción, es decir, «la aplicación de la maquinaria a los procesos de construcción, supone dificultades que no están presentes en otras industrias»; pero, aseguraba, «una técnica muy superior» podía ofrecer soluciones (Torroja, 1959: 3). Esta técnica superior se llamó «ciencias de la construcción», una amalgama de cálculo matemático, estadística, física,

11 Estos nuevos modos de producción fueron de importancia central para las relaciones de los ingenieros con sus respectivas sociedades políticas (Hughes, 1990). Los componentes estructurales de hormigón pretensado requerían que el resto de la estructura pudiera alojarlos. Era por tanto necesaria la coordinación tecnológica (para este concepto, ver Schmidt y Werle, 1997) y la compatibilidad funcional (para este concepto, ver de Vries, 2006).

EMPIRIA. Revista de Metodología de Ciencias Sociales. N. ${ }^{\circ}$ 18, julio-diciembre, 2009, pp. 85-114. ISSN: 1139-5737 
química y experimentación ${ }^{12}$. Los que hablaban de «ciencias de la construcción» no ponían excesivo énfasis en reivindicar la unidad de una nueva ciencia, sino en dejar patente que sus juicios expertos tenían fundamento teórico y experimental. Recordemos que en los institutos de investigación de la Europa de posguerra, las distinciones «entre investigación fundamental, desarrollo técnico y la invención de artefactos y técnicas se hacía cada vez más borrosa» (Pestre, 1997: 71).

Y, una vez más, el hormigón pretensado era el material idóneo para el desarrollo y expansión de las ciencias de la construcción: «con el pretensado, la Construcción se separa claramente de ese carácter crudo que solía diferenciarla de otras técnicas» (Torroja, 2004: 75). Para no romper el acero o el hormigón en el proceso de pretensado, se requerían cálculos complejos y operaciones precisas, lo que acercaba esta técnica al campo en el que Torroja mismo labró su reputación científica: las rupturas estructurales del hormigón. Torroja compartía cátedra en la Escuela de Caminos con Peña Boeuf. Éste último, académico de las ciencias desde 1934, dedicaba sus lecciones al hormigón como elemento llamado a 'racionalizar' el paisaje español transformándolo en un terreno productivo (Peña, 1953). «Desde el punto de vista hidráulico, España es desgraciadísima...Es un paisaje estepario, que causa desolación ¡Qué distinto de ese riente panorama que se aprecia desde la altura del resto de Europa!» (Peña, 1954, 229-235). Incidiendo en la conexión de la investigación científica y al estandarización, Peña Boeuf había sido el responsable de, como Ministro de Obras Públicas, preparar a principios de 1940 la Instrucción española para el hormigón armado (Peña, 1954: 227). Torroja, a su vez, enseñaba teoría de estructuras. En 1944 el discurso que pronunció con la ocasión, tan significativa para este apartado, de su nombramiento como miembro de la Real Academia de las Ciencias Exactas, Físicas y Naturales, llevaba por título «Unificación de teorías de tensión y deformación de hormigón armado en una solución probabilística para la teoría de la ruptura» (Torroja, 1944).

El principal colaborador de Torroja en el proyecto de Punto Azul era Alfredo Paéz, miembro del ITCC especializado en el comportamiento del hormigón pretensado. En la década de los cincuenta, Torroja y Páez colaboraron para establecer cálculos sobre elementos estructurales de este material (Páez y Torroja, 1951 y 1954). El problema principal al que se enfrentaban, establecer un coeficiente de seguridad que determinara las cargas máximas de un elemento de pretensado, estaba estrechamente unido a la adopción de estándares de rendimiento mínimo. Páez mostró que la teoría clásica de la elasticidad no era suficiente para analizar el comportamiento del acero y el hormigón tras el preten-

${ }^{12}$ Las primeras menciones a las «ciencias de la construcción» que he encontrado provienen de Italia principios del siglo XX (Almansi, 1901). Picon ha estudiado los orígenes institucionales de la conexión entre ingeniería y ciencia en el siglo XIX (Picon, 1992: 469-589). El sintagma «ciencias de la construcción» quedó finalmente plasmado en el nombre del ITCC en la década de los ochenta, cuando cambio por el de Instituto Eduardo Torroja de las Ciencias de la Construcción.

EMPIRIA. Revista de Metodología de Ciencias Sociales. N. ${ }^{0}$ 18, julio-diciembre, 2009, pp. 85-114. ISSN: 1139-5737 
sado, dado que, entre otras cosas, había que incluir la variable «tiempo», causante de la fatiga estructural, no sólo para las presiones finales en la obra sino también para las ejercidas en el taller (Páez, 1958). Para Torroja este incremento de variables suponía un doble espaldarazo para el ingeniero, pues además de dotar a su labor de mayor complejidad técnica le daba más libertad en el cálculo, y por tanto en el diseño (Torroja, 1958). Se comprende que desde la perspectiva de Torroja y Páez el hormigón pretensado era clave en dotar a la construcción de estatuto científico o, dicho de otro modo, de dar a sus investigaciones científicas relevancia político-económica en España.

\section{La norma provisional}

Estas tres características propias del hormigón pretensado — su afinidad a la prefabricación y, en particular, a la producción en masa, las oportunidades que ofrecía a la estandarización y su vinculación con el proyecto de las ciencias de la construcción - hacían de este material el candidato más apropiado para el impulso de la industrialización de la construcción que se pretendía con Punto Azul. Torroja proponía el hormigón pretensado como material más eficiente y económico para todas aquellas estructuras que requiriesen evitar la fractura o ser impermeables, como presas, acueductos, viaductos, tanques de presión, depósitos, etc. El pretensado resistía mejor que el hormigón armado la fatiga producida por vibraciones de grandes máquinas, incluidos trenes. Las estructuras laminares, aquellas por las que Torroja era conocido, también podían sacar partido al material. Pero para que esta expansión se produjese, el pretensado debía primero superar las dificultades que se le presentaban en sus usos más comunes, y Punto Azul sería clave para ello.

Para poner en marcha el servicio, lo primero que había que hacer era definir los estándares de mínimos. Dado que, en España, el uso del nuevo material había prendido especialmente en las viguetas, los técnicos del ITCC (en colaboración, como se explicará luego, con la Asociación Española de Hormigón Pretensado) prepararon una Norma provisional para viguetas de hormigón pretensado. La Norma contenía indicaciones sobre el número de técnicos por fábrica (asunto importante sobre el que volveremos en el último aparatado), sobre la definición de tipos de viguetas, la calidad y rendimiento mínimo de los materiales empleados (por ejemplo, tipos y pureza química del acero y cómo tratarlo, o del hormigón y sus componentes, como el agua y el cemento), las características de los elementos (diámetro del cable de acero y tamaño de las viguetas según carga; cálculos de resistencia de acuerdo a la variable tiempo, a la estructura y las funciones de ésta), y sobre la fabricación y uso en obra (moldes, anclajes, transporte de los materiales, almacenamiento). Por último, la Norma también especificaba qué tipos de ensayos se requerían para cada tipo de vigueta (AEHP, 1959). Punto Azul serviría para echar a andar esta Norma provisional y ayudar a ajustarla hasta establecer una definitiva. 
«El hormigón pretensado demanda industrialización», a decir de Torroja (Torroja, 1958). Y, por supuesto, la industrialización requería expertos investigadores en la construcción como los que el ITCC podría ofrecer. Los técnicos del ITCC transformarían el caos en orden. Torroja, Páez y Peña Boeuf tenían un programa científico y social cuando promovían el uso del hormigón. La industrialización y la estandarización, por tanto, no eran simplemente resultados naturales del desarrollo de la construcción. Los expertos del ITCC se consideraban a sí mismos como sujetos activos con la responsabilidad de mejorar la construcción haciéndola científica e industrializándola al modo de otras ramas de la producción.

\section{ESTÁNDARES INTERNACIONALES E INTEGRACIÓN TECNOLÓGICA EUROPEA}

El alcance de Punto Azul no era sólo nacional: «este servicio, preparado de acuerdo a las especificaciones y sistemas usados en otros países, establece las bases para un intercambio internacional de carácter técnico, siguiendo el espíritu actualmente dominante en el mundo occidental y, en particular, en Europa ${ }^{13}$. Para sus impulsores, la industrialización que Punto Azul promovía significaba acercar la construcción española a las nuevas tendencias que se desarrollaban en otros países, especialmente europeos. Veremos que la relación con estos países por parte de los ingenieros del ITCC no era de mera recepción pasiva, sino de una implicación activa que permite conectarla con procesos de integración española en Europa dados a escalas más ambiciosas.

Tras la Guerra Civil, Torroja dedicó la mayor parte de sus esfuerzos a la promoción del proyecto de industrialización de la construcción. Este proyecto económico-político contenía, como hemos visto, a las 'ciencias de la construcción' como uno de sus momentos esenciales, y esto incluía una constante relación con los avances en materias relacionadas que ocurrían en otros países. Por tanto, Torroja se preocupó de utilizar sus contactos y buena reputación — la cual queda patente en las palabras que le dedicó Frank Lloyd Wright: «el mejor ingeniero vivo»- para estrechar los vínculos de la construcción española con el exterior.

Así, Torroja fue el único delegado español en la reunión de 1948 de International Association for Bridge and Structural Engineering (IABSE), de la que fue nombrado asesor técnico en 1951. En 1954, año en el que Torroja recibió el primero de cuatro doctorados Honoris Causa en universidades de fuera de España, la IABSE celebró su congreso en el recién estrenado laboratorio del ITCC, Costillares, el cual mereció las alabanzas de los asistentes por estar «diseñado de acuerdo a los más modernos estándares» (Thürliman, 2000: 51-52). La actividad internacional de Torroja incluyó al ITCC en numerosas ocasiones,

13 AHT/N/A/043/001, «Borrador informativo con relación al Punto Azul y que se preparó para el Excmo. Ministro de la Vivienda», 1960.

EMPIRIA. Revista de Metodología de Ciencias Sociales. N. ${ }^{\circ}$ 18, julio-diciembre, 2009, pp. 85-114. ISSN: 1139-5737 
como, por ejemplo, la organización en 1950 de una reunión en Moscú del Consejo Internacional de la Edificación y la soviética Academia de la Construcción y la Edificación, una gestión que fue descrita por el ITCC como una mediación entre el Este y el Oeste (ITCC, 1960). Coincidiendo con la construcción del laboratorio Costillares en 1953, el ITCC publicó un número especial sobre más de treinta laboratorios de investigación de la construcción de todo el mundo (VVAA, 1953). Torroja animó activamente a la constitución de nuevas organizaciones internacionales, especialmente con colegas europeos, como el International Meeting of Laboratories of Research on Materials o la International Association for Shell Structures, cuya reunión fundacional se celebró en 1959 en Costillares, por ser, entre otras cosas gracias a los modelos que en ella se construían, «una institución única en el mundo para la investigación en nuevas estructuras, especialmente laminares» (Isler, 2003, 49).

El momento elegido por Torroja para el impulso de organizaciones de este tipo era especialmente propicio en una Europa que estaba en plena reconstrucción de posguerra y en los albores del boom económico. La cooperación científica y técnica en el continente se impulsó enormemente tras la Segunda Guerra Mundial; John Krige ha mostrado la importancia que en este proceso tuvo la hegemonía estadounidense, que proveía no solamente recursos económicos, sino ideología y aptitudes para la organización y la integración, incluyendo especialmente instituciones científicas y técnicas (Krige, 2006; Brenner, 2006). Por supuesto, el papel de los Estados Unidos hubiera sido en vano sin la colaboración de los diferentes países europeos, cada uno de los cuales se acercaba a esta incipiente cooperación técnica desde distintas situaciones e intereses.

En el caso de España, diferentes familias políticas percibían de modo diverso estos procesos de integración (Crespo, 2000). El régimen de Franco fue sancionado tras la Segunda Guerra Mundial mediante el aislamiento político. En la época de la Comunidad Europea del Carbón y del Acero (CECA) y de la Comunidad Económica Europea (CEE), establecidas en 1951 y 1957 respectivamente, el aislamiento político no siempre iba paralelo al aislamiento económico. Según Fernando Guirao, desde mediados de la década de los cincuenta la política oficial del régimen español era de «europeización»y, recíprocamente, Inglaterra y otros países europeos trataban a España como un miembro de hecho de la CECA, y esto no solamente en lucha con el comunismo - como en el caso de los sonados apoyos de Estados Unidos al régimen de Franco-, sino por necesidad económica para la reconstrucción de posguerra (Guirao, 1998: 189-206). La integración económica de España en Europa quedó patente con su integración de España como miembro asociado en la Organización Europea para la Cooperación Económica (OECE) en 1956, pero venía ya siendo ensayada desde finales de la década de los cuarenta para preservar los resultados de importantes acuerdos bilaterales, especialmente a raíz de la participación española en los primeros acuerdos de comercio europeos para agricultura, el Green Pool de la primera mitad de los años cincuenta (Guirao, 1995). 
Se suele vincular el inicio de la integración económica de España en Europa al informe de la OECE de 1959, muy crítico con la política económica del régimen, así como al Plan de Estabilización de la misma época; éste también se considera el punto de inflexión clave para las políticas científicas y técnicas del régimen (Sanz: 1997, 128-136). Sin embargo, la estrategia española de ignorar el aislamiento por parte de organizaciones políticas y centrarse en las económicas era anterior a esa fecha, y afectaba también a la integración técnica; por ejemplo, favoreciendo el ingreso en las incipientes organizaciones de estandarización. Así, España no ingresó en la ONU hasta 1955, y sin embargo formaba parte de la International Organization for Standardization (ISO) desde 1951. Siguiendo la introducción al presente artículo, no debiera minusvalorarse la importancia política de estas instituciones técnicas. La dimensión internacional de las ciencias las convierten en terreno idóneo para la historia de las relaciones internacionales (Wise, 1995; Porter, trust). La literatura histórica no ha dedicado excesiva atención a las organizaciones técnicas y científicas internacionales; sólo recientemente diversos historiadores de las ciencias y las técnicas han llamado la atención sobre la importancia de tenerlas en cuenta a la hora de escribir la historia de la integración europea, acuñando el término «integración tecnológica europea» (Misa y Schot, 2005; van der Vleuten y Kaijser, 2006).

La integración técnica europea se daba en el contexto de reconstrucción tras la Segunda Guerra Mundial, lo cual afectaba de modo inmediato al sector de la construcción. En aquellos años, «afortunadamente, las ciencias y técnicas de la construcción no esta[ba]n sujetas a las mismas restricciones y secretos que otras actividades, lo que permitía el libre flujo de información entre países» ${ }^{14}$.

Por supuesto, las organizaciones internacionales de estandarización servían a muy distintos intereses y sus resultados no siempre eran armónicos. Muchas de las creadas en Francia surgieron en el territorio ocupado por los nazis durante la Segunda Guerra Mundial (Tate, 2001); terminada ésta, tales organizaciones, algunas dedicadas a la construcción, se preservaron con vistas a asegurar el liderazgo francés en la reconstrucción europea como respuesta a la creciente producción alemana (Barjot, Baudouï y Voldman, 1997: 37).

Otras organizaciones internacionales surgieron ya acabada la guerra, incluídas las dedicadas al hormigón: la Fedération Internationale de la Précontrainte (FIP) y el Comitée Européen du Betón (CEB; hoy, ambas están fundidas en la FIB), fundadas en 1951 y 1953 respectivamente. La primera se fundó bajo la presidencia de Freyssinet, arropado por la francesa Association Scientifique de la Précontrainte con la intención de reunir a industriales y técnicos (AEHP, 1951). La segunda apareció bajo los auspicios del constructor francés BalencyBéarn con ánimo de unificar la investigación y aplicaciones referentes al hormigón armado (Levi, 2003: 24). Hay que señalar que Jupp Grote y Bernard Ma-

14 AHT/OI/A/001/076, Félix González, «D. Félix González escribe a D. Ynzenga, Presidente de la Agrupación de Fabricantes de Cemento», Octubre 1948.

EMPIRIA. Revista de Metodología de Ciencias Sociales. N. ${ }^{\circ}$ 18, julio-diciembre, 2009, pp. 85-114. ISSN: 1139-5737 
rrey enfocan la historia del hormigón pretensado desde la perspectiva de las relaciones europeas, con importantes resultados relativos al uso y desarrollo del pretensado en la Segunda Guerra Mundial, pero su análisis se detiene en 1945 y no contempla el papel de los países de Europa del sur (Gorte y Marrey, 2000 y 2003).

Torroja y Páez estuvieron vinculados a la FIP y al CEB desde sus inicios. Torroja fue miembro fundador de ambas, vicepresidente del CEB y, tras varios años en la vicepresidencia de la FIP, sustituyó a Freyssinet en su presidencia en 1958, cuando el idioma español se convirtió, junto al francés, al inglés y al alemán, en una de las lenguas oficiales de la Federación, que tenía miembros de cuarenta y dos países (VVAA, 1958). Poco después, Páez ocupó la vicepresidencia de la FIP (Piñero, 1959). Ambos dedicaron sus esfuerzos en el exterior a promover la estandarización mediante la creación de normas comunes para los diferentes países. El hormigón pretensado se estaba convirtiendo en un material importante para la inmensa labor de reconstrucción en diversos países de Europa, cuando la escasez de otros materiales, como el hierro, impulsaba el uso de elementos estructurales de materiales nuevos. Pero esta rápida expansión implicaba, al igual que en España, todas las incertidumbres propias de la falta de experiencia. La búsqueda de criterios para elaborar normas fiables era generalizada. Por tanto, en 1955 la FIP acogió la propuesta del ingeniero belga André Paduart para unificar las normas de los diversos países y le encomendó un informe sobre las diversas normas nacionales existentes. Para tal efecto, la FIP le asignó dos ayudantes, uno de ellos Páez (Paduart, 1958).

Paralelamente, Páez presentó en los sucesivos congresos de la FIP sus trabajos sobre la necesidad de establecer un coeficiente de seguridad nuevo para el pretensado, dada la insuficiencia de las teorías válidas para otros materiales. De este modo, logró que su propuesta de cálculo para el nuevo coeficiente, o «momento (flector) tope», fuera adoptada por el CEB, cuya instrucción para el hormigón armado fue seguida por la FIP en la elaboración de la suya para el pretensado (Levi, 2003: 25). El CEB también adoptó, en su reunión de Madrid de 1956, la «parábola de Madrid», o curva nomográfica, desarrollada por Torroja en base a ensayos realizados con modelos en Costillares, para representar la distribución de los esfuerzos en las zonas de comprensión (Páez, 1953).

En consecuencia, partes de la Norma provisional elaborada por el ITCC y que estaba previsto que Punto Azul ensayara fueron adoptadas por sucesivas versiones provisionales de la normal internacional elaboradas por la FIP y por la definitiva de 1970. Punto Azul era un modo de conectar la construcción nacional a estos desarrollos internacionales. Se concebía como el primer paso para un proceso de estandarización de la construcción europea en el que España tendría un papel activo. La expresión más ambiciosa de este proyecto fue el Agrément, una iniciativa del Centre Scientific et Technique du Bâtiment realizado con vistas a la armonización de las diferentes técnicas constructivas existentes en Europa y sobre el que Italia y el Benelux habían mostrado interés. Punto Azul pondría a 
España a la cabeza de este programa de integración técnica europea. ${ }^{15} \mathrm{Al}$ mismo tiempo, desde el punto de vista de los directivos del ITCC, esta actividad internacional legitimaba el proyecto doméstico de industrialización de la construcción, como se apreciará en las controversias en torno a Punto Azul a las que se dedica el siguiente apartado.

\section{EL ESTADO REGULADOR, O DE CÓMO CONTROLAR LA INDUSTRIA PRIVADA}

La historiografía de la ciencia suele situar en la Segunda Guerra mundial la alianza definitiva entre la investigación científica y el Estado. Iniciativas militares, tales como el proyecto Manhattan para la construcción de la bomba atómica, pusieron en manos de científicos e ingenieros ingentes sumas de dinero; la llamada Gran Ciencia fue un resultado de la guerra (Hughes, 1990; Sánchez Ron, 2007). Terminada la guerra, el documento entregado por Vannevar Bush al presidente de Estados Unidos, Franklin D. Roosevelt, exponía la idea embrionaria de los actualmente llamados programas de Investigación y Desarrollo, que fue exportada a Europa junto con los paquetes de ayuda norteamericanos dirigidos no sólo a reconstruir la belicosa Europa sino a transformarla (Block, 1977: 32108; Krige, 2006). La nueva situación supuso también un hito para las relaciones de los programas de investigación científica con la industria privada, que hoy han alcanzado cotas de identificación (Wise, 2006).

La colaboración entre la investigación científica y el interés industrial iba a menudo mediada por el Estado. De entre las muchas estancias en las que esto ocurrió, la más estudiada ha sido el llamado por el presidente norteamericano Dwight D. Eisenhower 'complejo militar-industrial,' al que habría que añadir la universidad: tras la Segunda Guerra Mundial «el mundo de la política, el ejército, la industria y la universidad estaban unidos más orgánicamente que nunca» (Pestre, 1997: 74; ver también los ensayos en Galison y Helvy, 1992). En marcado contraste con el caso anterior, el uso por parte de los Estados de la investigación científica como medio de controlar a la industria privada ha recibido mucha menor atención. Sin embargo, la historia del interés estatal por las ciencias y las técnicas ha tenido tanto que ver con la regulación, y en particular con la estandarización, como con las necesidades militares. Simon Schaffer cita las palabras del físico decimonónico James Clerk Maxwell, que celebraban los estándares —en este caso 'fundamentales', es decir, los que definen magnitudes o términos con los que las ciencias operan - como 'tesoros nacionales' que 'el hombre de negocios requiere por mor de la justicia, el hombre de ciencia por

15 AHT/N/A/043/001, «Informe que sobre las actividades del ITCC se presenta al pleno del Patronato Juan de la Cierva»: 7-8. 1960. En 1960 se fundó la Union Européenne pour l'Agrément technique dans la construction (UEAtc), que mediante el Euroagrément concede hoy en día Documentos de Idoneidad Técnica.

EMPIRIA. Revista de Metodología de Ciencias Sociales. N. ${ }^{0}$ 18, julio-diciembre, 2009, pp. 85-114. ISSN: 1139-5737 
mor de la verdad, y al Estado corresponde asegurarse de que nuestras... mediciones se mantengan estables» (Schaffer, 1995: 136). Desde los tiempos de Maxwell, parcelas de la realidad cada vez más amplias han sido sometidas al escrutinio de la estandarización; por ello, los procesos de formación de estándares son un terreno muy fértil para el estudio de las relaciones de los ingenieros, el Estado y el interés comercial (Rusell, 2006: 7).

Desde luego, la implicación del Estado en la estandarización no sólo afectó a científicos y tecnólogos, sino a la concepción misma de las funciones del Estado. Los años de la Europa de posguerra vieron el fortalecimiento del Estado como regulador de productos, y no meramente regulador económico. Según Peter Lundgreen, la implicación del Estado en la regulación de productos estuvo ligada a su creciente papel como consumidor -incluyendo a países de tradición 'liberal' (Edgerton, 2006)—, que justificaba la inversión en instituciones de ensayo y estandarización (Lundgreen, 1986: 19). Los institutos de investigación aceptaron de buen grado su nuevo papel de proveer a sus industrias nacionales con las herramientas técnicas necesarias para sobrevivir en una situación de competencia económica internacional, puesto que éste no sólo les aseguraba fondos para sus actividades sino que otorgaba relevancia político-económica a los desarrollos de sus disciplinas científicas. La regulación suponía, para científicos e ingenieros, una herramienta especialmente apta para asegurar la relevancia de sus hallazgos fuera del laboratorio.

De modo parecido a sus homólogos europeos, para los técnicos del ITCC la regulación era una vía abierta para la penetración de sus investigaciones en la sociedad española; es decir, para hacer efectivo su programa económico-político de industrialización de la construcción. Complementariamente a la popularización de las «ciencias de la construcción» mediante publicaciones y a la realización de proyectos propios para la industria pública y privada, establecer estándares de peso legal suponía un paso clave para la implementación de la investigación científica y técnica del ITCC en España. El cemento y sus derivados, sobre todoel hormigón pretensado, eran materiales especialmente adecuados para esta tarea.

Respecto al cemento, en España se producía en régimen de oligopolio, debido sobre todo a las limitaciones de su transporte, que hacían posible el control por parte de unas pocas grandes factorías de sus respectivos mercados regionales. Tras la Guerra Civil, la demanda de cemento se multiplicó anualmente (Coll y Sudriá, 1987). Para asegurar la producción mínima, evitar la escasez artificial y controlar la subida de los precios, el nuevo régimen creó en 1941 la Delegación Nacional para la Industrial del Cemento. No ha de sorprender que Torroja ocupara la dirección de este órgano, al que correspondía el control de la producción, de la distribución y de los precios. Aunque las relaciones del régimen de Franco respecto a la industria privada fueron a menudo más restrictivas que en otros países del entorno, en el caso del cemento el resto de países europeos tenían legislaciones de control similares (San Román, 1999; Bianchi, 1982). Es más, el control gubernamental era a menudo más nominal que otra cosa, dado el poder 
político — cuyas consecuencias para Punto Azul se tratarán más abajo- que a los productores de cemento otorgaba la urgencia con la que se demandaba el material (Rosado, 1997).

En cuanto al hormigón pretensado, dadas la falta de experiencia en su producción y uso y la complejidad técnica que requería, parecía proporcionar un terreno idóneo para extender las facultades reguladoras existentes a los productos. Torroja confiaba en que la industria aceptase voluntariamente esta regulación, pero estaba tan convencido de su necesidad que no descartaba imponerla de modo descendente, es decir, mediante la coacción del Estado. Para la primera estrategia, Torroja se apoyaba en la situación de debilidad creciente de los pequeños fabricantes de elementos estructurales de pretensado, que era causa tanto de la creciente competencia proveniente del aluminio como del desprestigio que los accidentes producían en la imagen del pretensado. Técnicos del ITCC actuaban como peritos expertos en juicios sobre accidentes, a veces fatales, causados por la inexperiencia en la fabricación y en el uso del pretensado. Esta inseguridad en torno al uso del nuevo material producía, a su vez, el aumento de peticiones de ensayo que, como se dijo al comienzo de este artículo, amenazaba con desbordar las capacidades del ITCC. Desde el punto de vista del ITCC, la solución a ambos problemas, la inseguridad y las excesivas pruebas de elementos individuales, pasaba por la regulación de estándares.

La solución propuesta, además, serviría para afianzar el ascendiente del ITCC sobre la empresa privada. En 1959, había en España más de 200 fábricas de viguetas de hormigón pretensado (AEHP, 1959: 5). Aunque muchas tenían laboratorios para ensayos propios, no todas podían costearse equipamientos ni personal cualificado para hacer estos ensayos fiables; es más, no tenían referente con el que comparar sus productos. Por ello, la Norma que Punto Azul promovería era para el ITCC una herramienta idónea para su penetración en la economía política española. Recordemos que la primera instrucción de la Norma Provisional exigía a las fábricas contar con personal técnico especializado, presumiblemente educado por los propios técnicos del ITCC. Esto, unido a la división del trabajo que la industrialización implicaba, aseguraría que las empresas privadas se convertirían en brazos por los cuales el ITCC podía hacer realidad sus planes de industrialización de la construcción española y de racionalización del paisaje español. La estandarización serviría para simplificar las relaciones del ITCC con la empresa privada, y la regulación para asegurar que los resultados de las «ciencias de la construcción» se distribuían por todo el territorio (para aspiraciones similares de científicos e ingenieros de otros países, Scott, 1998).

Ya en 1949, Torroja fundó la Asociación Española de Hormigón Pretensado (AEHP), adjunta al ITCC. La AEHP reunía a técnicos con fabricantes y usuarios, incluidos representantes del Sindicato Vertical del Vidrio y la Cerámica y del Gremio de Hormigón Prefabricado ${ }^{16}$. A mediados de los cincuenta, la AEHP

16 AHT/N/A/043/001, José A. Martino (Gremio de Prefabricados de Hormigón), «Carta a Jaime Nadal (ITCC)», 28 de octubre de 1960.

EMPIRIA. Revista de Metodología de Ciencias Sociales. N. ${ }^{o}$ 18, julio-diciembre, 2009, pp. 85-114. ISSN: 1139-5737 
solicitó al ITCC un servicio para ensayar y certificar sus productos de hormigón pretensado, en especial las viguetas de hormigón pretensado, por ser las más usadas, a la espera de que se preparara una Norma para su fabricación y uso. Para responder a esta solicitud había que preparar unas normas provisionales: la AEHP estableció una comisión encargada de las instrucciones para la fabricación de viguetas y otra para su uso; el ITCC se encargó de su unificación y de la redacción final de la Norma provisional. Para que ésta tuviese efecto, debía establecerse algún modo de certificar que se había seguido, con objeto de que quien ignorase los nuevos estándares quedase excluido del mercado. A este fin, Torroja presentó Punto Azul en una reunión de la AEHP en 1959, como servicio para certificar los elementos fabricados en serie que habían seguido la Norma (ITCC, 1959). En abril de 1960 se aprobaron los estatutos de Punto Azul en una reunión a la que acudieron unos 70 fabricantes, la Oficina de la Propiedad Industrial aprobó el logotipo, y en mayo se publicitó a los fabricantes de viguetas ${ }^{17}$. Pronto, las viguetas de un fabricante de Orense quedaron adornadas con el primer se1 lo azul ${ }^{18}$. Pese a todos estos esfuerzos, Punto Azul no sobrevivió el invierno.

Según Theodore Porter, los grupos profesionales que han presionado para la implantación de reglas numéricas objetivas lo han hecho, a menudo, como defensa ante una situación de debilidad; mientras tanto, grupos que no necesitaban este tipo de protección han sido más reacios a adoptar estándares numéricos (Porter, 1995). En nuestro caso, los pequeños fabricantes de elementos estructurales de hormigón pretensado, ante la competencia de otros materiales y el descrédito del suyo, accedieron a someterse a los estándares que proponía el ITCC; sin embargo, fabricantes mayores, de establecido prestigio y mercado, se opusieron con fuerza a la nueva norma. Los conflictos venían de atrás. Se mencionó más arriba que Torroja había logrado la imposición de un canon por venta a los fabricantes de cemento y derivados; la contrapartida es que el ITCC trabajaría para el beneficio de la industria. Sin embargo, los directivos del ITCC se veían como servidores al 'interés nacional' y a las 'ciencias de la construcción,' antes que a intereses particulares, lo que producía enfrentamientos con los empresarios ${ }^{19}$.

Así, cuando en 1948 el ITCC propuso una Norma para el Cemento Pórtland ajustada a otras europeas, los fabricantes, liderados por Federico Ynzenga (director de Cementos Pórtland, SA y presidente de la Asociación Nacional de Fabricantes de Cemento), se opusieron con argumentos contundentes: «la Norma será buena para países desarrollados con libertad de comercio — aunque incluso para esos países afecta a demasiados tipos de cemento-. Pero en España sería

17 AHT/N/A/040/001, AEHP, «Servicio de Comprobación de Elementos de Construcción "Punto Azul", Estatutos» 27 de abril de 1960.

18 AHT/N/A/041/001, «ITCC a Viguetas García», 27 septiembre 1960.

19 AHT/OI/A/001/023, Fernández Ladreda, J.M, Jímenez, A., Jimeno, E. y Torroja, E., «Informe de la ponencia sobre el dictamen presentado al Consejo Técnico-Asesor Permanente, por la Comisión Técnica Especializada del Cemento», 1946.

EMPIRIA. Revista de Metodología de Ciencias Sociales. N. ${ }^{\circ}$ 18, julio-diciembre, 2009, pp. 85-114. ISSN: 1139-5737 
desastrosa para el futuro del Cemento Pórtland. Si el cemento Pórtland se interviene fuertemente y al resto no, habrá una explosión de pastas más o menos efectivas que reclamarán el nombre de 'cemento' $»^{20}$. Jaime Nadal, impulsor de aquella norma (y que, años después, sería sucesor de Torroja al frente del ITCC tras el fallecimiento de éste), lamentaba que «el mayor error de la técnica nacional fue no cambiar las Instrucciones...no aceptar los ensayos y control de la producción. Cuando mencionábamos el asunto, siempre encontrábamos indiferencia,...oposición, o retrasos sutiles» (Nadal, 1959).

Estos mismos fabricantes del cemento y sus derivados que habían presentado batalla a la Norma para el cemento Pórtland volvieron al combate respecto al hormigón pretensado. La Norma provisional preparada por la AEHP era, desde su punto de vista, demasiado estricta, de modo que los fabricantes españoles perderían competitividad frente a los internacionales. Para preparar una Norma más adecuada a su punto de vista, el mismo Ynzenga, junto con otros fabricantes, fundó la Asociación Técnica para los Derivados del Cemento (ATDC). Cuando se lanzó Punto Azul, la ATDC se opuso al servicio frontalmente, alegando que, por su implantación, el ITCC se excedería en sus funciones, pues confundiría su labor con la publicidad comercial para determinadas firmas. Punto Azul debería limitarse a ser un organismo de consulta para pequeños y medianos productores, perdiendo el poder regulador que implica la extensión de certificados.

Los promotores de Punto Azul se defendieron: «Punto Azul no tiene interés comercial directo, dado que simplemente refleja resultados objetivos de carácter técnico omitiendo los aspectos sujetos a opinión, tales como las estadísticas económicas y otros $»^{21}$. Los fabricantes opositores no admitían los estándares técnicos del ITCC, por lo que rechazaban esta pretensión de objetividad. Para dar mayor legitimidad a Punto Azul, sus promotores se apoyaron en la dimensión internacional de los trabajos de Torroja y Páez: «no sería lógico ni justo que al preparar las Normas nacionales se olvidaran esos éxitos internacionales, que llenan de orgullo a la técnica Nacional y a nuestros prestigiosos especialistas» (Piñero, 1959: 36). La búsqueda de legitimidad para los proyectos nacionales estaba en la raíz de muchas de las actividades internacionales de los investigadores de la posguerra Europea (Krige y Preste, 1992). Sin embargo, este argumento también se volvió en contra de Torroja y Páez por dos razones. En primer lugar, la ATDC adujo que Punto Azul no servía para las condiciones españolas, carentes de acero y de cemento de calidad suficiente, lo que obligaría a la importación y, por tanto, el encarecimiento; la Norma era realista para países más ricos de Europa occidental, pero aceptarla haría peligrar la industria nacional —ni que decir tiene que-, en opinión de los técnicos del ITCC, los recursos españoles no justificaban esa actitud, sino que eran suficientes para implantar las normas de

${ }^{20}$ AHT/OI/A/002/098. Federico Ynzenga, «Carta de Cementos Portland al IC: Nota de reparos al proyecto de nuevo Pliego de Condiciones», 1948.

${ }^{21}$ AHT/N/A/043/001, «ITCC a la Junta Permanente del Patronato Juan de la Cierva», 1960.

EMPIRIA. Revista de Metodología de Ciencias Sociales. N. ${ }^{\circ}$ 18, julio-diciembre, 2009, pp. 85-114. ISSN: 1139-5737 
calidad propuestas. En segundo lugar, en las organizaciones internacionales del pretensado se reproducían parecidas diferencias entre los constructores y productores y los ingenieros investigadores (Levi, 2003: 25) ${ }^{22}$.

Torroja intentó buscar la solución al conflicto en la fuerza del Estado. También en esto decía estar siguiendo el modelo europeo: «Francia ha sido capaz de hacer lo que ha hecho porque el Estado consideró el problema interesante, a pesar de la oposición y pesimismo de bastantes industriales y técnicos(...). El Estado necesita impulsar y dirigir la especialización, al menos en sus primeros momentos de su desarrollo» (Torroja, 1958: 32-33). Desde el punto de vista de Torroja, se había demostrado que las empresas privadas se centraban demasiado en el interés particular e inmediato, prefiriendo soluciones torpes, perezosas y egoístas al consejo experto de los investigadores. Sólo el control del Estado tenía la independencia, el tiempo, y el dinero necesarios para impedir que la actitud suicida de los productores les hiciera quedarse atrás respecto a los avances de las «ciencias de la construcción» ${ }^{23}$.

Para forzar la intervención estatal, Torroja y Nadal contactaron con varios organismos oficiales, como el Ministerio de Industria y el Ministerio de la Vivienda. Sin embargo, no fueron siquiera recibidos. Las razones fueron, principalmente, dos. Por un lado, el peso político de los fabricantes de cemento y derivados del que ya hemos hablado antes. Por otro el creciente centralismo de las decisiones ejecutivas desde el nuevo gobierno de 1957, que incluían la coordinación de los proyectos científicos y técnicos como los de normalización de cara a cumplir las exigencias de Investigación y Desarrollo interpuestas por la OECE (Herrero, 1983: 359-571) ${ }^{24}$. El Patronato Juan de la Cierva fue el encargado de coordinar una Comisión para la Investigación Científica y Técnica. El Patronato encargó al Instituto de Racionalización centralizar las diferentes normativas técnicas bajo Una Norma Española (UNE). En estas circunstancias, y a pesar de que Torroja era miembro activo del Instituto de Racionalización, una iniciativa como Punto Azul encerraba ambigüedades respecto a su papel incompatibles con la UNE, lo que dio especial fuerza a las acusaciones de interés comercial que Punto Azul recibió. En efecto, antes de ordenar la definitiva cancelación de Punto Azul, el Patronato Juan de la Cierva suprimió una de sus características medulares, la capacidad de certificar series de productos, con el argumento de que: «el Instituto, en sus servicios de ayuda técnica, tiene que limitarse a certificar que una pieza determinada satisface las condiciones técnicas que el Instituto especifique; cualquier otra intervención, tales como las im-

${ }^{22}$ La ATDC era miembro del Bureau Internátional du Betón Manufacturé (actualmente, CEMBUREAU), pero no de la FIP, demostrando así las diferencias entre organizaciones internacionales dominadas por industriales y las dominadas por técnicos.

23 AHT/N/A/043/001, «Informe que sobre las actividades...» 1960.

${ }^{24}$ El que Torroja no fuera ajeno a este proceso, como coordinador de dos de las comisiones normalizadoras del Instituto Nacional de Racionalización, incluida una sobre «ensayo de materiales», no pareció bastar para poner de acuerdo a los diferentes órganos de la administración en torno a Punto Azul.

EMPIRIA. Revista de Metodología de Ciencias Sociales. N. ${ }^{\circ}$ 18, julio-diciembre, 2009, pp. 85-114. ISSN: $1139-5737$ 
plicadas en Punto Azul, competiría con otras oficinas de la Administración y no sería adecuada a la naturaleza de los institutos del Patronato» ${ }^{25}$.

El fracaso de Punto Azul marcó el principio del fin de los días de gloria del ITCC. Aunque ha seguido siendo un centro de referencia desde entonces, su época dorada acabó en la década de los sesenta, al principio de la cual falleció el profesor Torroja. En su conocido testamento, Torroja culpaba a «los de arriba» de incomprensiones y faltas de apoyo que impidieron a él y a sus colaboradores desarrollar todos sus proyectos e iniciativas; en carta a Antonio Suanzes fue más explícito: el problema de «la gente de arriba» es que se comportan como burócratas, causando «muchos de los problemas de España ${ }^{26}$. Seguramente, Punto Azul era una de las frustraciones que el profesor tenía en mente. En efecto, el centralismo de los nuevos modelos normativos y de la imposición de las regulaciones chocó con el intento de Torroja de establecer un método propio de certificado y ensayo de la industria privada. No es que los nuevos tecnócratas desplazaran a los viejos políticos, sino que técnicos con diferentes proyectos políticos pudieron más que los técnicos del ITCC y su entorno.

\section{CONCLUSIÓN}

Punto Azul acarreaba un triple proyecto de industrialización de la construcción, de integración tecnológica y científica con Europa y de regulación y control de la industria privada. Punto Azul fracasó, pero estudiar su breve historia ofrece una mejor comprensión no solamente de la normalización del hormigón pretensado, sino, sobre todo, del papel de la ciencia y la ingeniería en España y en el resto de la Europa de posguerra.

Las controversias en torno a Punto Azul nos ponen ante científicos muy activos en la España de Franco. Esto contradice algunas concepciones, aún vivas, del régimen como una losa monolítica bajo la cual los científicos e ingenieros trabajaban. Más que trabajar bajo tal losa, científicos e ingenieros intentaban conformarla a su gusto, promoviendo, en nuestro caso, la industrialización, la regulación y la integración con otros países. Esto no significa que el autoritarismo y la jerarquización no estuvieran muy presentes en las actividades de los investigadores, sino que no podemos juzgar a priori que esto producirá resultados contrarios al desarrollo científico o técnico (Scott, 1998: 85-181; Aly y Heim, 2002; Saraiva y Wise, en prensa).

Más en particular, la flexión política de los años 1957-1960, que corresponde a grandes rasgos con la breve historia de Punto Azul, queda matizada cuando se mira a la luz de este proyecto. Simplificando, la historia política de esos años puede resumirse como la entrada en el poder de unos tecnócratas que se

25 AHT/N/A/043/001, «Nota referente a los ensayos...», 23 de octubre 1960.

${ }^{26}$ Instituto Nacional de Industria, Archivo SEPI, Madrid. 0000040161, Eduardo Torroja (sin fecha), «Carta a Antonio Suanzes».

EMPIRIA. Revista de Metodología de Ciencias Sociales. N. ${ }^{0}$ 18, julio-diciembre, 2009, pp. 85-114. ISSN: $1139-5737$ 
ocuparon de liberalizar la economía y hacer así posible el 'milagro español'. Cuando se analiza el detalle del funcionamiento de capas de la sociedad política no restringidas al régimen de gobierno, este punto de inflexión no aparece como tal, sino como una curva continua con respecto a la década de 1950. En primer lugar, como señala el propio líder 'tecnócrata' Laureano López Rodó los diversos gobiernos de Franco habían contado con muchos ministros técnicos de ideología 'tecnocrática' desde los inicios del régimen (López, 1990: 100-01). En cualquier caso, como hemos visto en este artículo, la falacia consiste en pensar que tal ideología es unitaria y que los técnicos carecen de programa político. En segundo lugar, los historiadores económicos comienzan a considerar a la década de los cincuenta como necesaria para entender el posterior giro desarrollista ${ }^{27}$. Estudiar a agentes económicos vinculados al Estado pero normalmente ignorados por las historias económicas de más amplio alcance ofrece modos de entender esos continuismos en niveles tan básicos como el de las infraestructuras o los materiales de construcción.

Más allá de la historia española contemporánea, el estudio de Punto Azul permite 'seguir' al hormigón por sus viajes entre laboratorios de investigación, fábricas y países. El hecho de que los estándares permitan acercarse a tantos estratos diferentes de una sociedad política dada corrobora la importancia creciente que la historia de la ciencia otorga a los objetos fisicalistas, en tanto que estos permiten entender el funcionamiento de las ciencias en sus contextos más amplios. Además, estudiar estos procesos desde el punto de vista de un país autoritario y periférico ofrece nuevos campos a la historia de la ciencia, la historia de la integración europea y la de las organizaciones científico-técnicas de posguerra, por ejemplo en tanto que a ellas correspondió la función de proveer de herramientas al Estado para convertirlo en regulador de productos, y no sólo de mercados.

Es de esperar que más estudios atentos al detalle de cómo funcionaban de hecho las instituciones de investigación científicas y técnicas del primer franquismo contribuyan a la mejor comprensión histórica de ese período así como del la importancia de las ciencias y las técnicas para la historia moderna y contemporánea.

\section{BIBLIOGRAFÍA}

AEHP (Asociación Española de Hormigón Pretensado), 1951, «Conclusiones del congreso internacional del hormigón pretensado celebrado en Gante», Últimas avances técnicos en hormigón pretensado, $\mathrm{n} .{ }^{\circ} 9$.

\footnotetext{
27 Así, dice Fernando Guirao refiriéndose a la década de los 50 que «no había obstáculos exteriores claros; los historiadores de políticas económicas domésticas deberían aceptar el hecho de que, a pesar de una política económica obtusa, España formó parte de la Edad de Oro europea.» (Guirao, 1998: 203-05).
}

EMPIRIA. Revista de Metodología de Ciencias Sociales. N. ${ }^{\circ}$ 18, julio-diciembre, 2009, pp. 85-114. ISSN: $1139-5737$ 
AEHP (Asociación Española de Hormigón Pretensado), 1959, «Propuesta de normas para la fabricación y colocación de viguetas de hormigón pretensado», Ultimas noticias técnicas en estructuras de hormigon pretensado, n. ${ }^{\circ} 52$ (Sept.- Oct.), ITCC: 5.

ALDER, Ken (1997), Engineering the Revolution: Arms and Enlightenment in France, 1763-1815 (Princeton: Princeton University Press).

Almansi, E. (1901), Introduzione alla scienza delle costruzioni (Torino: C. Clausen).

Álvarez MuÑoz, Evaristo (2004), Filosofía de las ciencias de la tierra: el cierre categorial de la geología (Oviedo: Pentalfa).

Aly, Götz y HeIM, Susanne (2003), Architects of Annihilation : Auschwitz and the Logic of Destruction (Princeton: Princeton University Press).

ANDERSON, Charles W. (1970), The Political Economy of Modern Spain: Policymaking in an Authoritarian System (Madison: University of Wisconsin Press).

Arribas Macho, José María (1994), «Antecedentes de la sociedad de consumo en España: de la Dictadura de Primo de Rivera a la II República», Política y Sociedad, 16 (Madrid): 149-168.

Augustine, Dolores L. (2007), Red Prometheus: Engineering and Dictatorship in East Germany, 1945-1990 (Cambridge: MIT Press).

Azorin, Virtudes, SÁnchez-Montero, Yolanda, Villagra, Carlos (2005), «El Instituto de la Construcción y del Cemento: de la investigación científica a la innovación tecnológica», en Santiago Huerta (ed.), Actas del IV Congreso Nacional de Historia de la Construcción (Madrid: Instituto Juan de Herrera).

BArJot, Dominique, BAudouï, Rémi, VoldMan, Danièle (1997), Les Reconstructions En Europe 1945-1949 (Bruxelles: Editions Complexe).

BARNES, Barry y BloOR, David (1982), «Relativism, Rationalism and the Sociology of Knowledge», in Martin Hollis and Steven Lukes (eds.), Rationality and Relativism (Cambridge: The MIT Press): 21-47.

BianchI, Patrizio (1982), Public and Private Control in Mass Product Industry: The Cement Industry Cases, (The Hague: M. Nijhoff).

BIJKER, Wiebe E. (2005), «The Politics of Water. A Dutch Thing to Keep the Water Out or Not» in Bruno Latour and Peter Weibel (eds.), Making Things Public: Atmospheres of Democracy (Cambridge: The MIT Press): 512-529.

Blackbourne, David (2006), The Conquest of Nature: Water, Landscape and the Making of Modern Germany (Nueva York: W. W. Norton).

BLOCK, Fred L. (1977), The origins of international economic disorder: a study of United States international monetary policy from World War II to the present (Berkeley: University of California Press).

BLOOR, David (1999), «Anti-Latour», Studies in History and Philosophy of Science, vol. 30, 81-112.

BRENNER, Robert (2006), The Economics of Global Turbulence: The Advanced Capitalist Economies from Long Boom to Long Downturn, 1945-2005 (Londres: Verso).

BuENo, Gustavo (1972), Ensayo sobre las categorías de la economía política (Barcelona: La Gaya Ciencia).

Bueno, Gustavo, Primer ensayo sobre las categorías de las ciencias políticas (Logroño: Biblioteca Riojana, 1991).

Bueno , Gustavo (1992, 93), Teoría del cierre categorial (Oviedo: Pentalfa Ediciones).

CaHAn, David (1988), An Institute for an Empire: The Physikalisch-Technische Reichsanstalt 1871-1918, (Cambridge: Cambridge University Press). 
Carroll, Patrick (2006), Science, Culture, and Modern State Formation (Berkeley: University of California Press).

CAsinello, María J. (1997), «Concurso de viviendas experimentales, 1956: normalización, industria y arquitectura», en La vivienda experimental. Concurso de Viviendas Experimentales de 1956 (Madrid: Fundación Cultural COAM).

CAsinello, María J. (2000), «Razón científica de la modernidad española en la década de los 50», en José M. Pozo (ed.), Los años 50: la arquitectura española y su compromiso con la historia. Actas del II Congreso Internacional de Historia de la Arquitectura Moderna Española (Navarra: T6 Ediciones): 21-38.

ChANDler, Alfred D. y Hikino, Takashi (1990), Scale and Scope: The Dynamics of Industrial Capitalism (Cambridge: Belknap Press).

Coll Martín, Sebastián y Sudria, Carles (1987), El Carbón En España, 1770-1961: Una Historia Económica (Madrid: Turner).

CowAn, Henry J. (1978), Science and Building: Structural and Environmental Design in the Nineteenth and Twentieth Centuries (New York: Wiley).

Crespo Maclennan, Julio (2000), Spain and the Process of European Integration, 1957-85 (New York: Palgrave).

DE VRIES, Henk J. (2006), «Standards for Business: How Companies Benefit from Participation in International Standards Setting», in IEC Centenary Challenge (Geneva: IEC).

Edgerton, David (2006), Warfare State: Britain, 1920-1970 (Cambridge: Cambridge University Press).

Galison, Peter y Hevly, Bruce W. (1992), Big Science: The Growth of Large-Scale Research (Stanford: Stanford University Press).

Gallo, Max (1969), Histoire de l'Espagne franquiste (Verviers: Gérard \& co.).

Grote, Jupp y MARReY, Bernard (2000), Freyssinet, La précontrainte et l' Europe (Paris: Linteau).

Grote, Jupp y Marrey, Bernard (2003), «The Story of Prestressed Concrete from 1930 to 1945: A Step Towards the European Union», en Santiago Huerta (ed.), Proceedings of the First International Congress on Construction History (3 Vols. Madrid: Instituto Juan de Herrera).

GuIRAO, Fernando (1995), «Spain and the Green Pool: Challenge and Response, 1950 to 1955» en Richard T. Griffiths y Brian Girvin, The Green Pool and the Origins of the Common Agricultural Policy (Bloomsbury: Lothian Press).

GuIRAO, Fernando (1998), Spain and the Reconstruction of Western Europe, 1945-57: Challenge and Response (New York: St. Martin's Press).

HACKING, Ian (1983), Representing and Intervening : Introductory Topics in the Philosophy of Natural Science (Cambridge: Cambridge University Press).

Herrero CAStro, José Luis (1983), La introducción de la Organización Científica del Trabajo en las España de los años 40 y 50, 3 vols. (Bilbao: Universidad del País Vasco. Tesis doctoral sin publicar).

Huerga Melcón, Pablo (1999), La ciencia en La encrucijada: análisis crítico de la célebre ponencia de Boris Mijailovich Hessen, «Las raíces socioeconómicas de la mecánica de Newton», desde las coordenadas del materialismo filosófico, Oviedo: Pentalfa.

Hughes, Thomas P. (1990), American Genesis: A Century of Invention and Technological Enthusiasm, 1870-1970 (Nueva York: Penguin Books).

HunT, Bruce (1994), «The Ohm Is Where the Art Is: British Telegraph Engineers and the Development of Electrical Standards», Osiris 9 (2 $2^{\text {nd }}$ series, 'Instruments'): 48-63. 
ISLER, Heinz (2003), «Eduardo Torroja and the IASS, the International Association for Shell Structures», en Levi, F., Chiorino M. A. y Bertolini Cestari, C. (eds.), Eduardo Torroja: from the Philosophy of Structures to the Art and Science of Building (Milán: FrancoAngeli): 48-53.

ITCC (ed., 1959), Hormigón pretensado. III Asamblea General de la Asociación Española del Hormigón Pretensado. Primera Sesión (Madrid: Instituto de la Construcción y del Cemento).

KLEIN, Judy (2000), «Economics for a Client: the Case of Statistical Quality Control and Sequential Analysis», History of Political Economy Annual Supplement to Volume 32: 27-69.

KLEIN, Ronald (1995), Construing 'technology' as 'applied science': Public rhetoric of scientists and engineers in the United States, 1880-1945, Isis, 86: 194-221.

Kondratieve, Nikolai D. (1998), «Long Cycles of Economic Conjucture» (1926) en The Works of Nikolai D. Kondratiev, Vol.I. (Londres: Pickering \& Chatto Sollow).

Koyre, Alexandre (1983), Estudios De Historia Del Pensamiento Científico (Madrid: Siglo XXI).

Krige, John (2006), American Hegemony and the Postwar Reconstruction of Science in Europe (Cambridge: MIT Press).

KrIGE, John y Pestre, Dominique (1992), «Some Thoughts on the Early History of CERN» en Peter Galison y Bruce W. Hevley (eds.), Big Science. The Growth of Large-Scale Research (Stanford: Stanford University Press): 78-99.

KuHN, Thomas S (1962), The Structure of Scientific Revolutions. Chicago: University of Chicago Press.

Latour, Bruno (1988), The Pasteurization of France (Cambridge: Harvard University Press).

LATouR, Bruno (1999), «For David Bloor... and Beyond: a Replay to David Bloor's Anti-Latour», Studies in History and Philosophy of Science, vol. 30: 113-129.

LeVI, Franco (1951), Fluage, Plasticité, Précontrainte (Paris: Dunod).

LEVI, Franco (2003), «The Work of Eduardo Torroja: a «Milestone» in the Evolution of the Art of Building», en Levi, F., Chiorino M. A. y Bertolini Cestari, C. (eds.), Eduardo Torroja: from the Philosophy of Structures to the Art and Science of Building (Milán: FrancoAngeli): 24-30

LóPEZ RoDó, Laureano (1990), Memorias (Barcelona: Plaza \& Janes).

LÓPEZ GARCíA, S. (1994), El saber tecnológico en la política industrial del primer franquismo, (Madrid: Universidad Complutense. Tesis doctoral no publicada).

LundgreEn, Peter (1986), Standardization, Testing, Regulation: Studies in the History of the Science-Based Regulatory State (Germany and the U.S.A., 19th and 20th Centuries) (Bielefeld: Universität Bielefeld B. Kleine Verlag).

MisA, Thomas J. y SсHOT, Johan (2005), «Inventing Europe: Technology and the Hidden Integration of Europe», in History and Technology, (Vol. 21, n. ${ }^{\circ}$ 1, March 2005): pp. $1-19$

MoKYr, Joel (1990), The Lever of Riches: Technological Creativity and Economic Progress (Nueva York: Oxford University Press.

MoRADIELlos García, Enrique (200), La España de Franco (1939-1975): política y sociedad (Madrid: Síntesis).

NADAL, Jaime (1959), «Servicios del Instituto, en relación con las condiciones ténicas de la construcción en el momento actual», Informes de la Construcción, n. ${ }^{\circ} 116$ (December 1959).

EMPIRIA. Revista de Metodología de Ciencias Sociales. N. ${ }^{\circ}$ 18, julio-diciembre, 2009, pp. 85-114. ISSN: 1139-5737 
O'Connell, Joseph (1993), «The Creation of Universality by the Circulation of Particulars», Social Studies of Science, vol. 23, n. ${ }^{\circ} 1$ (Feb, 1993): 129-73.

ORDÓNEZ, Javier y SÁNCHEZ-Ron, José Manuel (1996), «Nuclear Energy in Spain: From Hiroshima to the Sixties» en Paul Forman y José M. Sánchez Ron, National Military Establishments and the Advancement of Science and Technology: Studies in 20th Century History (Boston: Kluwer Academic Publishers).

PADUART, André (1958), «Bases d'un règlement international concernant le béton précontraint», en FIP, Third Congress of the FIP, Berlin 1958, Papers (London: Cement and Concrete Association): 220-27.

PÁEZ, Alfredo y TORROJA, Eduardo (1951), Nuevo método de cálculo del hormigón pretensado (Madrid: Instituto Tecnico de la Construccion y del Cemento).

PÁEZ, Alfredo (1953), Intervención en First Congress of the FIP, London 1953, Papers (London: Cement and Concrete Association): 131-32.

PÁEz, Alfredo y TORROJA, Eduardo (1954), «Set and Reinforced Concrete» en M. Reiner, (ed.), Building Materials: their elasticity and inelasticity (Amsterdam: North-Holland Publishing Company): 290-361.

PÁEZ, Alfredo (1958), «Esfuerzos cortantes después de la figuración» en FIP, Third Congress of the FIP, Berlin 1958, Papers (London: Cement and Concrete Association): 66-79.

PeÑa BoEuf, Alfonso (1953), «Un siglo de hormigón armado en España», Revista de Obras Públicas, 2857 (101, Vol. 1, Número especial del centenario): 23-34.

PeÑa Boeuf, Alfonso (1954), Memorias de un ingeniero político (Madrid: Estades).

Pestre, Dominique, «Science, Political Power and the State» in John Krige \& Dominique Pestre (eds.) Science in the Twentieth Century, (Amsterdam: Harwood Academic Publishers, 1997): 61-76.

Picon, Antoine (1992), L'invention De L'ingénieur Moderne: L'ecole Des Ponts Et Chaussées, 1747-1851 (Paris: Presses de l'Ecole nationale des ponts et chausses).

PIÑERO (1959), «Informe sobre la IV Asamblea General de la Asociación Española de Hormigón Pretensado», Ultimas noticias técnicas en estructuras de hormigón pretensado, n. 53 (Nov.-Dec. 1959).

PORTER, Theodore M. (1995), Trust in Numbers: The Pursuit of Objectivity in Science and Public Life (Princeton: Princeton University Press).

Presas i PuIG, Albert (2005), «Science on the Periphery. The Spanish Reception of Nuclear Energy: An Attempt at Modernity», Minerva, 43 (2005): 197-218.

Roberts, Lissa, SchafFer, Simon y DeAR (2007), The Mindful Hand: Inquiry and Invention from the Late Renaissance to Early Industrialisation (Amsterdam: Koninkliijke Nederlandse Akademie van Wetenschappen).

Roca i Rosell, Antoni y SÁnchez Ron, José Manuel (1990), Esteban Terradas (18831950): Ciencia Y Técnica En La España Contemporánea (Barcelona: Instituto Nacional de Técnica Aeroespacial, Ediciones del Serbal).

RosAdo CuBERO, Ana Isabel (1997), La organización industrial del sector cementero español, 1942-1996 (Madrid: Universidad Complutense de Madrid. Tesis doctoral no publicada).

RuSELl, Andrew L. (2006), «Industrial Legislatures: the American System of Standardization», en IEC Centenary Challenge, (Geneva: IEC).

SAN ROMAN, Elena (1999), Ejército e industria: el nacimiento del INI (Barcelona: Crítica). SÁnchez Ron, José Manuel (1999), Cincel, martillo y piedra: historia de la ciencia en España (siglos XIX y XX) (Madrid: Taurus). 
SÁnCHEZ Ron, José Manuel (2007), El poder de la ciencia: historia social, política y económica de la ciencia (siglos XIX y XX) (2. ${ }^{\text {a } e d ., ~ B a r c e l o n a: ~ C r i ́ t i c a) . ~}$

SANTAS, Asier (2004), «1950: Una norma española, una arquitectura internacional», en J. M. Pozo e I. López Trueba (coords.), Modelos alemanes e italianos para España en los años de posguerra. Actas del IV Congreso Internacional de Historia de la Arquitectura Moderna Española (Navarra: T6 Ediciones): 123-136.

Santesmases, María Jesús (2001), Entre Cajal Y Ochoa: ciencias biomédicas en la España de Franco, 1939-1975 (Madrid: Consejo Superior de Investigaciones Científicas).

SANZ MENÉNDEZ, Luis (1997), Estado, ciencia y tecnología en España: 1939-1997 (Madrid: Alianza Editorial).

SAmbricio, Carlos (2004), Madrid, vivienda y urbanismo, 1900-1960: de la «normalización de lo vernáculo» al plan regional (Madrid, Akal).

SARAIVA, Tiago (2007), «Inventing the Technological Nation: The Example of Portugal (1851-1898)», History and Technology, 23:3, 263-273.

SARAIVA, Tiago y WiSE, Norton (en prensa), Genetics and Autarky, número especial de Historical Studies in the Natural Sciences (Berkeley: University of California Press).

ScHAFFER, Simon, (1995), «Accurate Measurement is an English Science», en Norton M. Wise (ed.), The Values of Precision (Princeton: Princeton University Press): 135-172.

SchmidT, Susanne y Werle, Raymund (1997), Coordinating Technology: Studies in the International Standardisation of Telecommunications (Cambridge: The MIT Press).

ScotT, James C. (1998), Seeing Like a State: How Certain Schemes to Improve the Human Condition Have Failed (New Haven: Yale University Press).

Slaton, Amy E. (2001), Reinforced Concrete and the Modernization of American Building, 1900-1930 (Baltimore: Johns Hopkins University Press).

TATE, Jay (2001), «National Varieties of Standardization», in Peter A. Hall and David Soskice (eds.), Varieties of Capitalism: The Institutional Foundations of Comparative Advantage (Oxford: Oxford University Press): 442-473.

ThÜRLIMAN, Bruno (2000), «Torroja visto desde el extranjero», en Emilio Alarcón et al., De la construcción a la ciencia. Ayer y hoy de Eduardo Torroja, (Madrid: Academia de Ingeniería).

Timmermans, Stefan y Berg, Marc (2003), The Gold Standard: The Challenge of Evidence-Based Medicine and Standardization in Health Care (Philadelphia: Temple University Press).

Torroja, Eduardo (1944), Discurso, leído en el acto de su recepción el día 29-11-44 (Madrid: Real Academia de Ciencias Exactas, Físicas y Naturales).

TorRoja, Eduardo (1958), «El hormigón pretensado y sus campos específicos de aplicación» en Últimos Avances Técnicos en Estructuras de Hormigón Pretensado, n. ${ }^{\circ}$ 9: $1-33$.

Torroja, Eduardo (1959), «Las actividades del Instituto de la Construcción y del Cemento», Informes de la Construcción, Vol. III, n. ${ }^{\circ}$ 27: 1-18.

TORROJA, Eduardo (2004), Razón y ser de los tipos estructurales (1958; Madrid: CSIC).

Usselman, Steven W. (2002), Regulating Railroad Innovation : Business, Technology, and Politics in America, 1840-1920 (Cambridge: Cambridge University Press).

VAN DER VleUtEN, Erik y KAIJSER, Arne (eds., 2006), Networking Europe: transnational infrastructures and the shaping of Europe, 1825-2000 (Sagamore Beach: Watson Publishing International).

EMPIRIA. Revista de Metodología de Ciencias Sociales. N. ${ }^{\circ}$ 18, julio-diciembre, 2009, pp. 85-114. ISSN: $1139-5737$ 
VVAA (1953), «Laboratorios de Investigación», Informes de la Construcción, n. ${ }^{\circ} 36$. VVAA (1958), Third Congress of the Fédération Internationale de la Précontrainte (London: FIP).

WINNER, Langdon (1986), «Do Artefacts have Politics?» in Winner (ed.), The Whale and the Reactor: A Search for Limits in an Age of High Technology, pp.19-39.

WISE, M. Norton (ed.; 1995), The Values of Precision (Princeton: Princeton University Press).

WiSE , M. Norton (2006), «Thoughts on the of Politicization of Science through Commercialization», Social Research, vol. 73, No. 4. (Winter 2006).

\title{
RESUMEN
}

Este artículo estudia la historia de un intento fallido de regulación de elementos de la construcción, en concreto de viguetas de hormigón pretensado, al final de la década de los cincuenta en España: Punto Azul. Punto Azul fue un proyecto concebido para el desarrollo y la implementación de estándares para elementos de la construcción producidos en serie. La iniciativa fue propuesta por el Instituto Técnico de la Construcción y del Cemento, dirigido por el ingeniero de fama internacional Eduardo Torroja, con vistas a reunir en torno a sus expertos tanto al capital privado como al Estado. Sin embargo, el proyecto tenía detractores que, tras intensas pugnas, lograron derrotarlo. Este hecho nos servirá para, tirando del hilo de una iniciativa de apariencia puramente técnica, desvelar la imbricación de las ciencias, las técnicas y la economía política en un período de cambios dentro del régimen franquista. Así, veremos que Punto Azul implicaba un programa de industrialización de la construcción que incluía el desarrollo de las 'ciencias de la construcción', una creciente integración con los incipientes organismos europeos y un proyecto de control y regulación de la industria privada. En la introducción y conclusión se examinan las implicaciones metodológicas del estudio.

\section{PALABRAS CLAVE}

Ciencia, economía política, estandarización, franquismo, hormigón pretensado.

\begin{abstract}
In this article, I explore the history of a failed attempt at standardization of prestressed concrete joists (small beams) at the end of the 1950s in Spain: Punto Azul (Blue Point). Punto Azul was a means of developing and spreading performance standards for mass-produced structural components. The project, championed by the Instituto Técnico de la Construcción y del Cemento (Tech-
\end{abstract}


nical Institute for Construction and Cement) and his director Eduardo Torroja, of international reputation as a structural engineer, tried to gather the state and private business around construction experts. However, the project aroused opposition that, after heated controversy, led to its termination. This paper will follow the Punto Azul story in order to unveil the intermingling of science, technology and political economy in a time of changes within the Francoist regime. Thus, I will show that Punto Azul conveyed a whole program of industrialization of construction that included the development of 'the sciences of construction', a growing technological integration with other European countries and a project of control and regulation of Spain's private industry.

\section{KEYWORDS}

Francoism, political economy, prestressed concrete, science, standardization. 